\title{
Pirazmonam Sodium
}

National Cancer Institute

\section{Source}

National Cancer Institute. Pirazmonam Sodium. NCI Thesaurus. Code C76564.

The sodium salt form of pirazmonam, a monocyclic beta-lactam antibiotic. Pirazmonam is more active than aztreonam against nonfermentative gram-negative bacteria. 